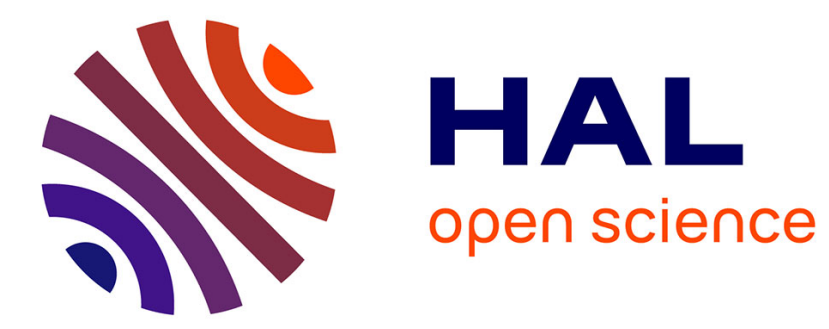

\title{
RESIDUAL APPROACH ON A HIERARCHICAL SEGMENTATION
}

\author{
Beatriz Marcotegui
}

\section{To cite this version:}

Beatriz Marcotegui. RESIDUAL APPROACH ON A HIERARCHICAL SEGMENTATION. IEEE

International Conference on Image Processing, Oct 2014, Paris, France. hal-01080417

\section{HAL Id: hal-01080417 \\ https://hal.science/hal-01080417}

Submitted on 5 Nov 2014

HAL is a multi-disciplinary open access archive for the deposit and dissemination of scientific research documents, whether they are published or not. The documents may come from teaching and research institutions in France or abroad, or from public or private research centers.
L'archive ouverte pluridisciplinaire HAL, est destinée au dépôt et à la diffusion de documents scientifiques de niveau recherche, publiés ou non, émanant des établissements d'enseignement et de recherche français ou étrangers, des laboratoires publics ou privés. 


\title{
RESIDUAL APPROACH ON A HIERARCHICAL SEGMENTATION
}

\author{
Beatriz Marcotegui \\ beatriz.marcotegui@mines-paristech.fr \\ MINES ParisTech, PSL Research University, CMM - Centre for Mathematical Morphology \\ 35 rue Saint Honoré - Fontainebleau, France
}

\begin{abstract}
Residual operators analyze the evolution of an image subject to the application of a series of transformations, for example a series of openings of increasing size. When a significant object is filtered out by a transformation corresponding to its size, an important residue is observed. Maximal residues are kept for each pixel, indicating the most significant objects present in the image. Different families of operators have been used in the literature: morphological openings or closings, attribute openings or openings by reconstruction. In this paper we propose to compute residues on a hierarchy of partitions, computing the differences between regions at different hierarchical levels based on the classical earth mover's distance. The advantage of our approach is that it is autodual and generic as it can be applied with any hierarchical approach.
\end{abstract}

Index Terms - Numerical residues, ultimate opening, waterfall, $\mathrm{P}$ algorithm, image segmentation, mathematical morphology, earth's mover distance.

\section{INTRODUCTION}

A residue is defined as the difference between two operators. Among residual operators we find the morphological gradient, the top-hat transformation, thinnings or the skeleton transform, all of them extensively used in the literature. More recently Serge Beucher generalized the residue of a family of operators to gray level images introducing the quasi-distance and the ultimate opening operators [1]. Previous versions of ultimate opening were independently proposed in the literature under the name of adaptive opening by Vogt [2] or morphological profiles by Pesaresi et al. [3, 4]. Successful applications based on these operators have been developed such as rock analysis[5], automatic text localization [6], façade segmentation [7] and remote sensing approaches [8, 9, 10].

In practice, residual operators based on morphological openings (or closings) or attribute openings with different attributes have been used. The choice of the attribute has a strong influence in the final result. Moreover those operators deal with openings or closings, focusing on either bright or dark objects. Some authors propose to combine both polarities choosing for each pixel the polarity leading to the highest contrast estimation $[4,11]$. Thus bright and dark objects are correctly processed but intermediate gray level regions can be missed. A similar idea is used by Maximally Stable Extremal Regions (MSER) [12]. The image is quantized with a given threshold step. A min-tree of the resulting image is built. The area difference between connected components of consecutive thresholds is computed. Significant regions are chosen when this area difference constitutes a regional minimum. The choice of the threshold step decides which are the regions to be compared and is a critical parameter of the algorithm.

In this paper we propose a residual approach based on a hierarchy, dealing with both polarities simultaneously and correctly considering intermediate gray level regions.

\section{HIERARCHY}

Let $H=\left\{P_{0}, P_{1}, \ldots P_{N}\right\}$ be a hierarchy of nested partitions of image $I . P_{0}$ corresponds to the finest partition: e.g the set of pixels of the input image, the watershed result (usually over-segmented) or any other input partition. $P_{N}$ corresponds to a partition with a single region for the whole image. $P_{i}$ is a simplification of $P_{i-1}$ which means that a region $R_{i}^{j}$ of $P_{i}$ is the union of a set of regions $R_{i-1}^{k}$ of $P_{i-1}$ such that $R_{i-1}^{k} \subset R_{i}^{j}$ :

$$
R_{i}^{j}=\left\{\cup R_{i-1}^{k} \mid \forall k R_{i-1}^{k} \subset R_{i}^{j}\right\}
$$

$R_{i}^{j}$ is said to be the parent of regions $R_{i-1}^{k}$ contained in it.

The proposed approach is general: any hierarchical approach can be used. In order to illustrate it we use wathershed-based hierarchies. The Watershed algorithm usually leads to an over-segmentation of the image. Several hierarchical approaches have been proposed to overcome this problem. In this paper we focus on waterfall hierarchy [13] and its enhancement, the P algorithm [14]. The waterfall [13] is a watershed-based hierarchical segmentation approach. It consists in two steps:

- first, each region is filled with the value of the lowest pass point of its frontier. The pass point is the gray level 
value of the pixel where, during the flooding process associated with the watershed, neighbouring "lakes" (regions) meet for the first time. A morphological reconstruction may be used for this purpose.

- second, the watershed of the resulting image is computed.

In the example of Figure 1 the watershed lines are indicated by arrows and only solid line arrows will be preserved by the waterfall. The process may be iterated until a single region covers the whole image, establishing a hierarchy among the frontiers produced by the watershed. An efficient graphbased waterfall algorithm is presented in [15].

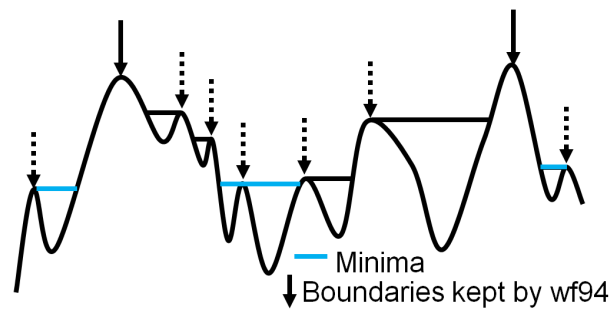

waterfall principle

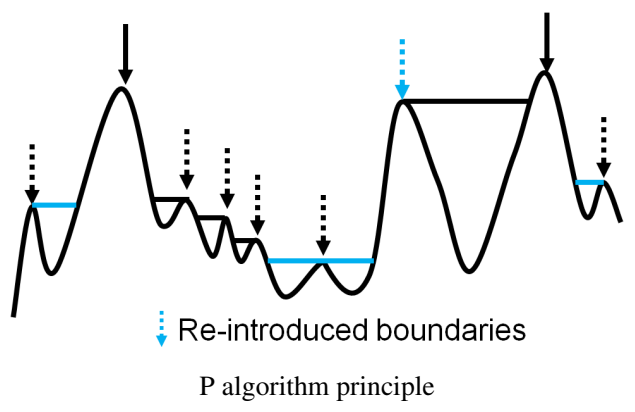

Fig. 1: Waterfall and P Algorithm principle.

Each waterfall step forces regions to merge with at least one neighbor. Thus, significant regions may be removed, if they have neighboring regions which have more hierarchical levels than themselves, due to texture for instance. P algorithm analyzes the boundaries removed by the waterfall and re-introduces those that are "compatible" with the remaining ones. The notion of compatibility is local, depending on the neighbor boundary values preserved by the waterfall. The process consists in the following:

- the minimum pass point of each waterfall catchment basin (MinPassPoint $\left.\left(C B_{i}\right)\right)$ is computed.

- all boundaries removed by the waterfall algorithm with a value $v$ close to the min pass point of its catchment basin are re-introduced in the partition. Close to the min pass point means that $\mid$ MinPassPoint $\left(C B_{i}\right)-$ $v \mid<v$ or in other words $2 * v>\operatorname{MinPassPoint}\left(C B_{i}\right)$.
In figure 1(b), dotted arrows are boundaries removed by the waterfall and blue dotted arrows are those re-introduced by $\mathrm{P}$ algorithm.

\section{RESIDUAL APPROACH ON A HIERARCHY}

Ultimate opening identifies important regions thanks to the strong gray level change they produce when they are filtered out by an opening of its corresponding size. In order to introduce the residual operator linked to a hierarchy, we have to estimate the distance between child and parent nodes within a hierarchy of nested partitions. The mean grey level difference is not a good choice because it is meaningless for the highest levels of the hierarchy. We propose to use a histogram distance computed between a region, $R_{i-1}^{k}$, and its parent, $R_{i}^{j}$ :

$$
\operatorname{Res}\left(R_{i-1}^{k}\right)=\operatorname{distance}\left(R_{i-1}^{k}, R_{i}^{j}\right)
$$

The Earth Mover's Distance (EMD) between two distributions is defined as the minimal cost that must be paid to transform one distribution into another. It is supposed to match perceptual similarity better than other distribution distances used for image retrieval [16]. The EMD avoids quantization and other binning problems typical of histograms and allows partial matching. When used to compare distributions with the same overall mass, the EMD is a true metric.

We use EMD in order to estimate the distance between regions of consecutive hierarchical levels. If the fusion is performed within a homogeneous region the $\operatorname{distance}\left(R_{i-1}^{k}, R_{i}^{j}\right)$ is low whereas this distance will be high if distinctive regions are merged. The idea is to detect significant changes in the hierarchy and avoid them, keeping the regions before critical mergings. Then, the hierarchical level is chosen locally, as residues are computed for each region of $P_{i-1}$. The decision is taken region by region. Thus the final partition will be made up combining different hierarchical levels.

The principle is illustrated in figure 2. In this example, the finest partition has 6 regions $(\mathrm{A}-\mathrm{F})$ and the hierarchy has 3 levels. The hierarchy is represented as a tree. The process starts from the finest partition. Residual image is initialized with the distance between leave regions and their parents. Then higher hierarchical levels are considered. The relevance of a region is estimated as the distance with its parent. Then children's and parent's relevances are compared:

- If children are more important than their parent, children are kept (regions A, B and C in our example).

- If the parent is more relevant than its children, the parent is chosen (regions are merged). This is the case for region $\mathrm{H}$, including regions $\mathrm{D}, \mathrm{E}$ and $\mathrm{F}$.

The process is iterated for higher hierarchical levels. In figure 2(b) plain nodes are kept while dashed ones are removed. Figure 2(c) illustrates the final result. Each time that a region 
$R_{i}^{k}$ is kept, its corresponding residue $\operatorname{Res}\left(R_{i}^{k}\right)$ is stored in the residual image $(T(p))$. The residual image $T(p)$ for a pixel $p$ is then defined as:

$$
T(p)=\max \left\{\operatorname{Res}\left(R_{i-1}^{k}\right) \mid p \subset R_{i-1}^{k} \text { for } i \in\{1, \ldots N\}\right\}
$$

$T(p)$ conveys contrast information of a region with its neighborhood.
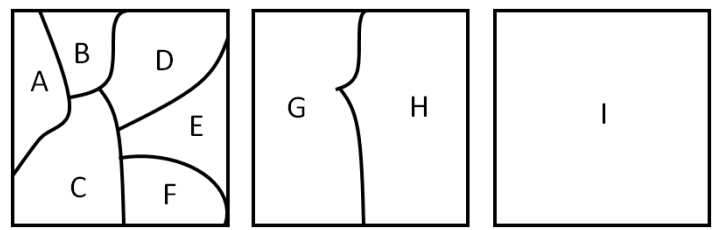

(a) Hierachy of nested partitions

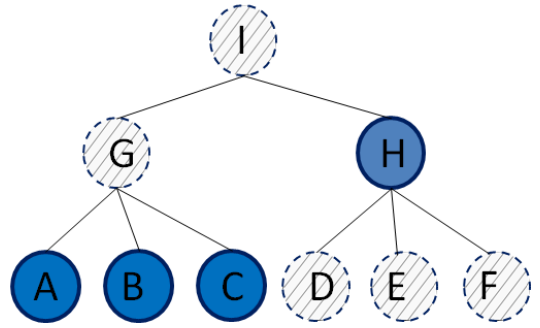

(b) Hierarchical tree

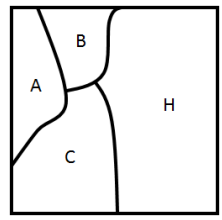

(c) Partition result
Fig. 2: Principle of residual approach on a hierarchical segmentation.

\section{RESULTS}

In this section the residual approach introduced in previous section is applied to real images. Commonly used filters, such as levelings [17], can applied prior to image segmentation for image simplication. Figure 3 shows the intermediate images of the process. Image 3(a) shows the original image and (b) the corresponding filtered image with a leveling of size 10 . The finest partition (c) corresponds to the result of a watershed applied to the gradient of the filtered image. Then the waterfall hierarchy, starting from the finest partition, is applied leading to four hierarchical levels illustrated in figures (d)-(f) (the last level being a single region for the whole image). The hierarchy is analyzed using the Earth Mover's Distance, as previously explained. The final partition is shown in figure (h) and the corresponding residual image is shown in figure (g). We can observe that significant regions are correctly selected from the hierarchy. We can argue that some regions are under-segmented. For example the blue square region on the bottom right part of the image 3(h) contains two significant regions. This region is over-segmented in $P_{1}$ (dark and light blue region in the upper part) while it is undersegmented in $P_{2}$ (dark green region). $P_{2}$ region has a higher residue, then under-segmentation is chosen.
In order to get a correct segmentation result, it is important to have significant regions at a given level of the hierarchy. It is known that waterfall produces few hierarchical levels, with strong simplifications between consecutive levels. P algorithm produces more hierarchical levels than the waterfall hierarchy, more meaningful partitions and a non-null partition at its highest level. In order to complete the hierarchy, the two last waterfall levels are added. Figure 4 shows successive P algorithm hierarchical levels. Figures $4(\mathrm{~h})$ and (i) show the final partition and the corresponding associated contrast. We can observe in $P_{3}$ the correct segmentation of the square area mentioned above, and correctly selected in the final partition 4(h).

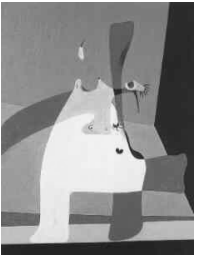

(a) Original

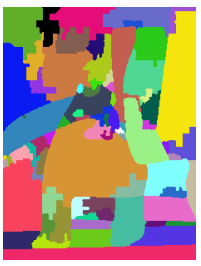

(d) wf $1\left(P_{1}\right)$

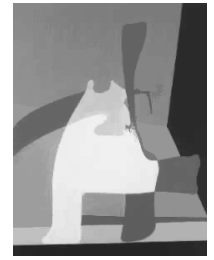

(b) Filtered Image

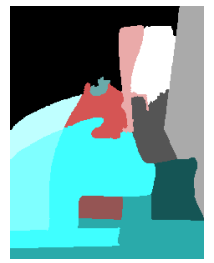

(e) wf $2\left(P_{2}\right)$

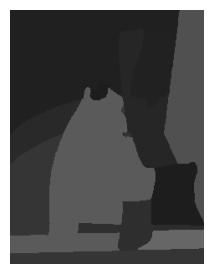

(g) $T(p)$ (contrast information)

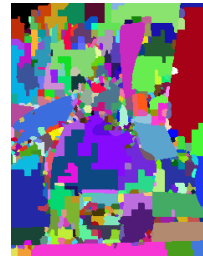

(c) Fineseg $\left(P_{0}\right)$

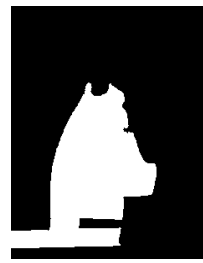

(f) wf $3\left(P_{3}\right)$

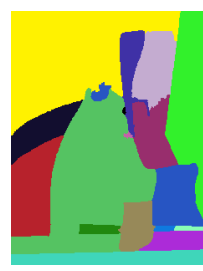

(h) Final Partition (Residual wf)
Fig. 3: Residual operator based on a waterfall hierarchy.

More examples comparing P algorithm, waterfall residual approach and $\mathrm{P}$ algorithm residual approach are provided in figure 5. We can observe that $\mathrm{P}$ algorithm residual approach outperforms $\mathrm{P}$ algorithm itself as well as waterfallbased residual approach.

\section{CONCLUSION AND PERSPECTIVES}

In this paper we generalize the residual approach to a segmentation hierarchy. We demonstrate that the residual approach is able to identify significant regions from the hierarchical segmentation approach. The number of regions adapts 


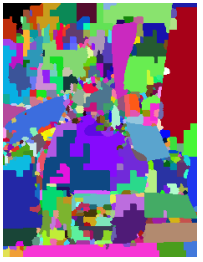

(a) Fineseg $\left(P_{0}\right)$

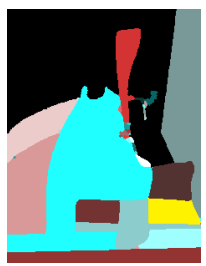

(d) algo $\mathrm{P},\left(P_{3}\right)$

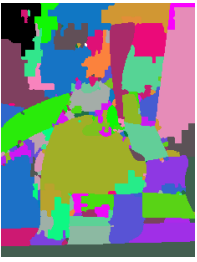

(b) algo $\mathrm{P},\left(P_{1}\right)$

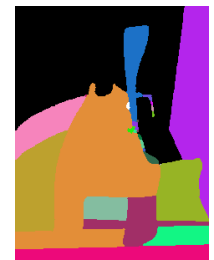

(e) algo $\mathrm{P},\left(P_{4}\right)$

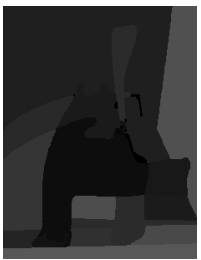

(g) $T(p)$ (contrast information)

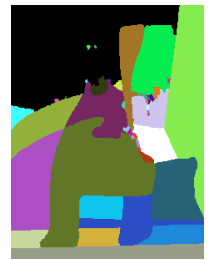

(c) algo $\mathrm{P},\left(P_{2}\right)$

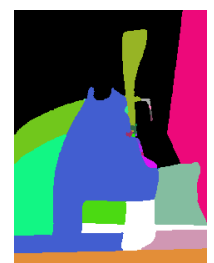

(f) algo $\mathrm{P},\left(P_{5}\right)$

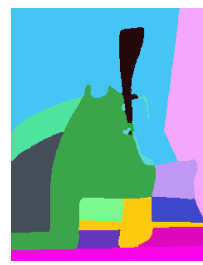

(h) Residual algo $\mathrm{P}$ result

Fig. 4: Residual operator based with on a P algorithm hierarchy.

automatically to the image complexity. Moreover, additional contrast information is provided. This contrast is based on the earth mover's distance. We show that $\mathrm{P}$ algorithm residual approach outperforms $\mathrm{P}$ algorithm itself as well as waterfall residual approach. In the future fast implementations of the proposed algorithm will be addressed and color distances will be considered.
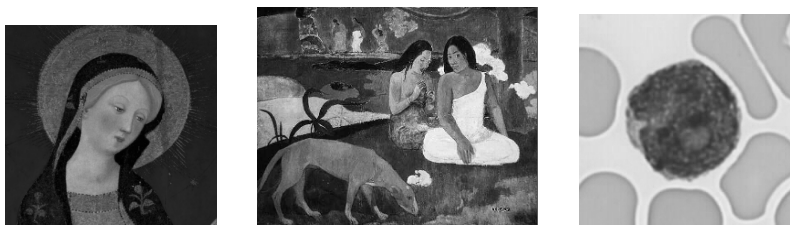

Original image
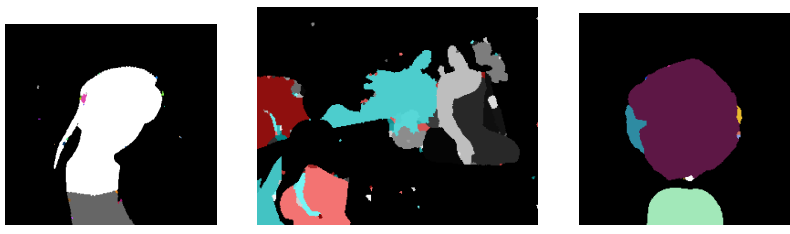

$\mathrm{P}$ algorithm resulting partition
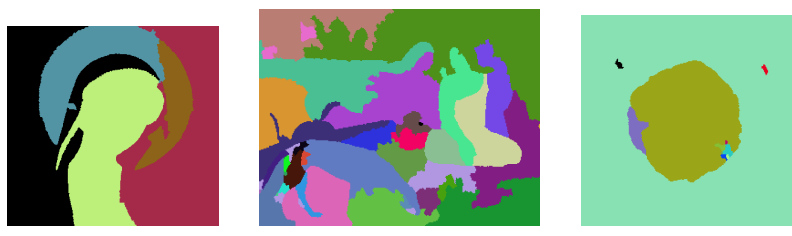

Waterfall-based residual segmentation
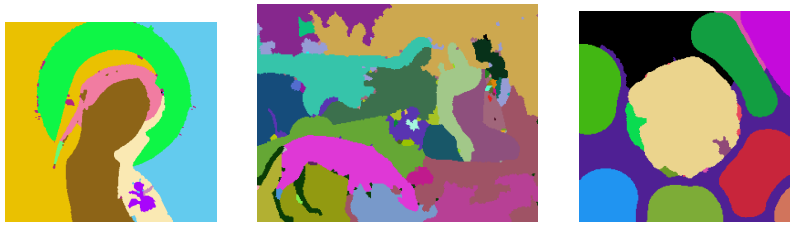

P Algorithm-based residual segmentation

Fig. 5: Segmentation results comparison 


\section{REFERENCES}

[1] Serge Beucher, "Numerical residues," Image Vision Computing, vol. 25, no. 4, pp. 405-415, 2007.

[2] R. Vogt, "A spatially variant, locally adaptive, background normalization operator," in Mathematical morphology and its applications to image processing, J. Serra and P. Soille, Eds., pp. 45-52. Kluwer Academic Publishers, 1994.

[3] Martino Pesaresi and Jon Benediktsson, "Image segmentation based on the derivative of the morphological profile," in Mathematical Morphology and its Applications to Image and Signal Processing, John Goutsias, Luc Vincent, and Dan S. Bloomberg, Eds., vol. 18, pp. 179-188. Springer US, 2000.

[4] M. Pesaresi and J.A. Benediktsson, "A new approach for the morphological segmentation of high-resolution satellite imagery," Geoscience and Remote Sensing, IEEE Transactions on, vol. 39, no. 2, pp. 309 -320, feb 2001.

[5] Souhail Outal and Serge Beucher, "Controlling the ultimate openings residues for a robust delineation of fragmented rocks," in The 10th European Congress of Stereology and Image Analysis, June 2009.

[6] Thomas Retornaz and Beatriz Marcotegui, "Scene text localization based on the ultimate opening," International Symposium on Mathematical Morphology, vol. 1, pp. 177-188, October 2007.

[7] Jorge Hernández and Beatriz Marcotegui, "Ultimate attribute opening segmentation with shape information," in Proceedings of the 9th International Symposium on Mathematical Morphology and Its Application to Signal and Image Processing, Berlin, Heidelberg, 2009, ISMM '09, pp. 205-214, Springer-Verlag.

[8] M. Pedergnana, P.R. Marpu, M. Dalla Mura, J.A. Benediktsson, and L. Bruzzone, "Classification of remote sensing optical and lidar data using extended attribute profiles," IEEE Journal on Selected Topics in Signal Processing, vol. 6, no. 7, pp. 856-865, 2012.

[9] J.A. Benediktsson, M. Pesaresi, and K. Arnason, "Classification and feature extraction for remote sensing images from urban areas based on morphological transformations," IEEE Trans. Geoscience and Remote Sensing, vol. 41, no. 9, pp. 1940-1949, September 2003.

[10] S. Lefevre, J. Weber, and D. Sheeren, "Automatic building extraction in vhr images using advanced morphological operators," Proc. IEEE/ISPRS Joint Workshop Remote Sensing and Data Fusion over Urban Areas, 2007, 2007.
[11] Jonathan Fabrizio and Beatriz Marcotegui, "Fast implementation of the ultimate opening," in Proceedings of the 9th International Symposium on Mathematical Morphology and Its Application to Signal and Image Processing, Berlin, Heidelberg, 2009, ISMM '09, pp. 272281, Springer-Verlag.

[12] J. Matas, O. Chum, M. Urban, and T. Pajdla, "Robust wide-baseline stereo from maximally stable extremal regions," Image and Vision Computing, vol. 22, no. 10, pp. 761-767, 2004, British Machine Vision Computing, BMVC 2002.

[13] Serge Beucher, "Watershed, hierarchical segmentation and waterfall algorithm," in Mathematical morphology and its applications to image processing, Jean Serra and Pierre Soille, Eds., pp. 69-76. Kluwer Academic Publishers, 1994.

[14] Serge Beucher and Beatriz Marcotegui, "P algorithm, a dramatic enhancement of the waterfall transformation.," http://hal-ensmp.archives-ouvertes.fr/hal00835019/PDF/P-Algorithm_SB_BM.pdf, 2009-09.

[15] B. Marcotegui and S. Beucher, "Fast implementation of waterfall based on graphs," in Proceedings of the 7th International Symposium on Mathematical Morphology and Its Application to Signal and Image Processing, April 2005, ISMM '05, pp. 177-186.

[16] Y. Rubner, C. Tomasi, and L. J. Guibas, "The earth mover's distance as a metric for image retrieval," International Journal of Computer Vision, vol. 40, no. 2, pp. 99-121, 2000.

[17] Fernand Meyer, "Levelings, image simplification filters for segmentation," Journal of Mathematical Imaging and Vision, vol. 20, no. 1-2, pp. 59-72, 2004. 